\title{
PORTAFOLIO DE MARCAS: Un marco conceptual*
}

\author{
Claude Chailan \\ PROFESOR DE CERAM SOPHIA-ANTIPOLIS, FRANCIA \\ GRADUADO EN CIENCIAS POLÍTICAS, ECONOMía Y GESTIÓN
}

André Boyer

PROFESOR DE LAS UNIVERSIDADES, NIZA SOPHIA-ANTIPOLIS, FRANCIA

PH. D. EN MANAGEMENT

Luis Felipe Calderón Moncloa

PROFESOR DE ESAN Y CANDIDATO DOCTORAL DE LA

UniVERsité DE LA MEDITERANEÉ, AIX MARSEILlE I, FRANCIA

\begin{abstract}
Resumen
En los últimos veinte años, el conocimiento de la marca se ha enriquecido considerablemente, a la par con la importancia que ésta ha adquirido. Pero hace falta profundizar en un aspecto de este tema: la relación entre las marcas de una misma empresa desde la perspectiva de la organización de sus respectivos territorios. Esta dimensión ha sido muy poco estudiada, aunque forma parte del día a día de los gerentes, que se interrogan sobre las estrategias de marca más pertinentes para alcanzar el éxito. Los trabajos metodológicos subrayan la necesidad de operacionalizar los conceptos para permitir su medición. La relación entre conceptos no observables (por ejemplo, desempeño y portafolio de marcas) se estima a través del estudio de la relación empírica entre ciertas dimensiones de estos conceptos. Así, encontrar las dimensiones mensurables de un concepto permite dar cuenta de éste y medir algunos de sus componentes. Este artículo propone operacionalizar el concepto de portafolio de marcas y destacar sus dimensiones mensurables.
\end{abstract}

\section{Introducción}

La marca es un tema recurrente en la investigación de marketing y objeto de numerosos trabajos y de una literatura abundante. Sin embargo, si bien la relación

* Trabajo presentado a la 38 asamblea anual del Consejo Latinoamericano de Escuelas de Administración, Cladea, llevada a cabo en Lima entre marcas ha sido ya largamente abordada dentro de la temática de la arquitectura de la marca, un aspecto de esta relación aún no ha sido estudiado en profundidad: la relación entre las marcas de una

del 21 al 24 de octubre de 2003 con el título «Gerencia: retos y nuevos paradigmas». 
misma empresa; y no desde el punto de vista de la relación jerárquica que determina su grado de subordinación, sino desde el punto de vista de la organización de los territorios de cada una de estas marcas. Esta problemática plantea la cuestión de una metadimensión de la marca: el portafolio de marcas.

Actualmente, el concepto de portafolio de marcas está en el corazón mismo de la reflexión de los dirigentes de las empresas más grandes del mundo. Así Alan Lafley, presidente de Procter \& Gamble comenta:

Actualmente nuestro portafolio de productos de limpieza para el hogar y los productos para bebes y de higiene femenina nos conviene. El objetivo es proceder a extensiones de gama alrededor de nuestras marcas faro, eventualmente por crecimiento externo. Por el contrario, queremos comprimir nuestro portafolio de productos de cuidado, belleza y salud [...]. Nuestro oficio es desarrollar las marcas que duren toda la vida o más ${ }^{1}$.

Por su parte, Lindsay Owen-Jones, presidente de L'Oréal indica:

Tenemos un portafolio de marcas bastante completo, equilibrado. Las adquisiciones eventuales serán, más que nada de sociedades que completarán nuestro portafolio de nichos o que completarán nuestro dispositivo internacional $^{2}$.

1. Entrevista aparecida en el diario económico Les Echos del 8 de enero de 2003 (traducción de los autores).

2. Entrevista aparecida en el diario Le Figaro del 21 de febrero de 2003 (traducción de los autores).
Estas preocupaciones hacen eco a cientos de otras similares, en un entorno cada vez más turbulento. Sin embargo, mientras que las investigaciones sobre la marca suscitan un vivo interés en nuestra disciplina, el estudio del portafolio de marcas todavía no se ha formalizado. Se trata, no obstante, de una etapa clave en la comprensión de la ventaja competitiva de numerosas empresas (Sharma, 1999; Slater y Olson, 2001) y del marketing estratégico (Day, 1994; Trinquecoste, 1999). En efecto, desplazando las fuentes de ventaja competitiva a un nivel superior (Juga, 1999; Reynaud, 2001), esta ventaja se hace más difícil de comprender y de imitar. La combinación de marcas dentro del seno de un portafolio parece corresponder a este movimiento.

Esta nueva problemática motiva al investigador a ensayar respuestas a cuestiones hasta ahora poco exploradas, como por ejemplo:

- ¿Qué razones conducen a una empresa a desarrollar o a no desarrollar un portafolio de marcas?

- ¿La existencia de un portafolio de marcas está ligada a un tamaño crítico, a una estructura geográfica o a un esquema particular de creación de valor?

- ¿Existen estrategias específicas de portafolios de marcas según cada mercado?

Las obras metodológicas (Evrard, Pras y Roux, 2000) subrayan la necesidad de operacionalizar los conceptos para permitir su medición. Por ello, antes de abordar el estudio de campo para contrastar nuestra hipótesis, parece esencial, en una primera fase del trabajo exploratorio, proce- 
der a la identificación de las principales dimensiones del concepto de portafolio de marcas, a fin de hacerlo mensurable (Reynaud, 2001).

El objeto del presente artículo es describir las condiciones para el surgimiento y evolución de los agrupamientos de marca con la finalidad de establecer las premisas de una investigación centrada en el portafolio de marcas. El artículo se propone identificar las principales etapas en la construcción de un portafolio de marcas y determinar la importancia estratégica de este concepto, para luego proponer una definición de éste.

Con este propósito, seguiremos la siguiente estructura: i) límites del territorio de marcas, ii) rol estratégico de los agrupamientos de marcas y iii) definición y dimensiones del portafolio de marcas.

\section{Límites del territorio de marcas}

\subsection{Evolución de la arquitectura de marca}

Un portafolio de marcas es resultante de un proceso de múltiples fases. El origen de este proceso está en la problemática del territorio de marcas (Kapferer, 1991), la que a su vez está ligada a la cuestión de la extensión de marca (Michel, 2000). Es necesario, por ello, dominar esta génesis para contextualizar el concepto de portafolio de marcas.

En un primer momento las empresas buscan maximizar el potencial de sus marcas a través del desarrollo de extensiones. Aparecen límites que implican una coexistencia de marcas para cubrir todos los segmentos de mercado deseados, lo que con- duce a un agrupamiento de marcas. Estos agrupamientos de marca necesitan ser gerenciados para ser óptimos, lo que da origen al concepto de portafolio de marcas.

El estudio de la marca puede ser analizado como una sucesión de fases que permiten situarla en una perspectiva histórica. Así, para Kapferer ${ }^{3}$ se pueden distinguir cinco etapas:

- Estudio del valor de la marca (Farqhar, 1990; Aaker, 1991; Kapferer, 1991; Keller, 1993; Krishnan, 1996).

- Explotación de este valor de la marca, en particular la extensión de la marca (Aaker y Keller, 1990; Broniarczyk y Alba, 1994; Michel, 2000).

- Arquitectura de la marca (Kapferer, 1991; Laforêt y Saunders, 1994).

- La relación con el consumidor; numerosas vías de investigación, en particular acerca de la proximidad a la marca (Alba y Chattopadhyay, 1986; Fournier, 1998), apego a la marca (Lacoeuilhe, 2000; Perrin-Martinencq, 2002), compromiso con la marca (Amine, 1998; Gurviez, 1999), la fidelidad (Jacoby y Chestnut, 1978; Dyson et al., 1996).

- Personalidad de la marca (Levy, 1959; J. L. Aaker, 1997; Ferrandi y ValetteFlorence, 2002; Caprara, Barbaranelli y Guido, 2002).

Un punto de vista complementario a esta perspectiva histórica, sugerido por Kapferer, consiste en examinar la marca

3. Jornada AFM Las Marcas, 12 de diciembre de 2002, conferencia de clausura. 
desde el ángulo de su «relación con... ». Podemos entonces distinguir las siguientes cuatro dimensiones principales abordadas en su investigación:

- La relación marca-producto (Sappington y Wernerfelt, 1985; Semprini, 1992; Kapferer, 2000).

- La relación marca-consumidor (Lancaster, 1966; Farqhar, 1990; Plummer, 2000; Lambin, 1989; McEnally y De Chernatony, 1999; Sicard, 2001; Azoulay, 2002).

- La relación marca-empresa (Aaker 1991; Laforêt y Saunders, 1999).

- La relación marca-marca (KaufmanOdin, 1997; Bensa y Ladwein, 1996; Michel, 2000).

Esta lectura cruzada y dinámica de los estudios de la marca permiten descubrir un eje de investigación que enriquece el conocimiento de la marca dentro de la última dimensión citada: la relación marcamarca. Nosotros nos centraremos en este último tipo de relación.

Diferentes trabajos han analizado esta dimensión de la relación entre marcas, sea desde el punto de vista externo, es decir, competitivo (dos marcas pertenecientes a dos empresas diferentes y que compiten en un mismo mercado), o desde el punto de vista interno, es decir, estudiando la relación jerárquica de una marca con otra en el seno de una misma empresa.

En la actualidad, sin embargo, la relación entre las marcas de una misma empresa rebasa estos modelos clásicos para orientarse hacia la coexistencia de marcas poderosas, cuyas relaciones deben o deberían ser reguladas: el portafolio de marcas.

\subsection{Límites a las extensiones de marca}

Una de las mayores consecuencias del incremento de los costos del marketing -y del riesgo asociado- es la búsqueda de una utilización extensiva de las marcas existentes. Esta utilización de las marcas existentes se opera ya sea en «modo menor», a través del desarrollo de nuevas líneas a fin de penetrar nuevos segmentos de mercado en una misma categoría de productos (Ladwein, 1993; Kotler, 1997), o en «modo mayor», a través de las extensiones de marca cuyo objetivo es capitalizar sobre las franquicias de marca para penetrar en nuevas categorías de productos (Aaker y Keller, 1990; Cegarra y Merunka, 1993).

A fin de evitar una elevada tasa de fracasos al lanzar nuevos productos, la estrategia de extensión de marcas es percibida -desde hace años- como una de las opciones de crecimiento más rentables para las empresas. La extensión de marca permite aprovechar la notoriedad y la imagen de una marca para penetrar nuevos mercados. El éxito de esta opción reside en la difundida opinión de que la utilización de marcas existentes para penetrar en nuevas categorías de productos conduce a una reducción sustancial de los costos de lanzamiento, incrementa la eficacia de las acciones de marketing, refuerza la probabilidad de aceptación de los nuevos productos y, por ende, aumenta también las probabilidades de éxito (Morrin, 1999).

Los trabajos experimentales (Milberg, 1993, citado por Michel, 2000) muestran cómo el interés de las extensiones de mar- 
ca cambia en función de los productos considerados. El apalancamiento que representa una marca puede reducir sustancialmente el riesgo vinculado a la introducción de un producto a un nuevo mercado, ofreciendo a los consumidores la proximidad y el conocimiento de una marca conocida. Pero también existe un riesgo de disminución del valor de este activo (Aaker y Keller, 1990). Una extensión inapropiada podría conducir a asociaciones deteriorantes que serían costosas o imposibles de corregir (Ries y Trout, 1981).

De manera general, optar por una extensión implica asumir un riesgo estratégico importante, que compromete recursos humanos, financieros y de tiempo. Interrogarse sobre la extensión de la marca es preguntarse acerca de los límites de esta extensión; es decir, la cuestión del territorio de la marca (Kapferer, 1996). La marca encuentra límites de varios tipos a su expansión.

Ciertas investigaciones se han focalizado en la relación entre ciertos elementos de la marca -o de su extensión-, en la formación de la actitud de los consumidores hacia el nuevo producto (Bottomley y Holden, 2001). La importancia de la adecuación entre la marca de origen y la categoría de extensión es puesta en evidencia por Aaker y Keller (1990), quienes resaltan cuatro principales factores determinantes tanto de las posibilidades de extensión de la marca como de la amplitud de las extensiones.

Estos factores son: la credibilidad de los atributos de la marca; la existencia de un vínculo entre las clases de producto de origen de la marca y las de la extensión; la capacidad de transferencia de la marca de origen; la complementariedad entre las clases de productos; y la percepción del consumidor sobre la dificultad para hacer la extensión (si la extensión es percibida como demasiada, es menos bien aceptada, pues es percibida como una «explotación» de la marca). Otros estudios se han interrogado sobre el posible aporte negativo de una extensión de marca hacia la marca madre (Loken y John, 1993; Gürhan-Canli y Maheswaran, 1998).

Morrin (1999) muestra que las extensiones de las marcas no dominantes tienen un efecto más importante sobre la memoria de los consumidores que las extensiones de marcas dominantes ${ }^{4}$, lo que puede explicarse por el hecho de que los consumidores «perdonan» a las marcas dominantes sus «desviaciones de mercado».

Pero muchos otros factores pueden indicar la mayor o menor aceptación de una extensión de marca por los consumidores. Podemos citar el número de las extensiones (Dacin y Smith, 1994; Morrin, 1999), el tiempo de exposición a las extensiones (Klink y Smith, 2001; Swaminathan, Fox y Reddy, 2001), la información proporcionada (Aaker y Keller, 1990; Klink y Smith, 2001), la motivación (Gürhan-Canli y Maheswaran, 1998), la dimensión innovadora del consumidor (Klink y Smith, 2001), y el espacio geográfico (Bottomley y Holden, 2001).

Para clarificar el concepto de límite de extensión de la marca, formalizaremos el conjunto de estos límites a través de tres

4. Herr, Farqhar y Fazio (1996) definen las marcas dominantes como aquellas que tienen tendencia a ser reconocidas primero cuando los consumidores son expuestos a una señal de una categoría de producto. 
ejes que recorren los principales trabajos efectuados sobre este tema, yuxtaponiéndose a la temática del territorio de marca:

- El territorio-mercado (Dacin y Smith, 1994; Loken y John, 1993).

- Aquellas que se deben al territorioconsumidores (Gürhan-Canli y Maheswaran, 1998; Klink y Smith, 2001).

- Y finalmente, aquellas asociadas al territorio temporal (Morrin, 1999; Bottomley y Holden, 2001).

El incremento de la competencia obliga a la empresa a manejar la multiplicación de productos, gamas y marcas. Se trata de definir un sistema de identidades destinado a manejar los atributos y articulaciones entre las marcas así como su posicionamiento (Ries y Ries, 2000). Este sistema permite a la firma construirse un capital de marcas perennes, que serán los vectores tanto para los productos existentes como para los nuevos productos.

Además, a estos límites consustanciales a la estructura del territorio de marca se sobrepone a menudo una voluntad positiva de yuxtaposición de marcas dentro de las empresas. Esta voluntad puede estar ligada a consideraciones de prudencia (no depender de una sola marca) o financieras (maximización de la creación de valor limitada por la demasiada dependencia de una marca). Tampoco debemos ignorar que dar demasiada autonomía a las áreas de investigación y desarrollo puede engendrar una extensión, si no incontrolada, al menos no planificada del territorio de marcas.

Muchas firmas se encuentran así con un conjunto más o menos coherente, más o menos dispar de marcas, algunas por adquisiciones sucesivas; mientras que otras habrán lanzado a lo largo del tiempo múltiples marcas para responder a la necesidad de diferenciación de los mercados, sin tomar en consideración la necesidad de ubicar estas marcas las unas respecto a las otras. Estos diferentes límites han conducido a las empresas a yuxtaponer, comprar o crear muchas marcas para superar estos frenos al desarrollo de la relación del fabricante con el consumidor (Riezebos, 2003). Las extensiones de marca están, pues, rodeadas por las restricciones que limitan el potencial del territorio de marca y devienen motivos, lo que conduce a una yuxtaposición de marcas en el seno de las empresas. En este contexto, la marca ya no es por ella misma un elemento suficiente de diferenciación o de desempeño superior. Es su acomodamiento en el seno de un grupo de marcas lo que le permite generar el crecimiento deseado por la empresa.

Producto de la convergencia de los límites del territorio de marca y de la voluntad de las empresas de responder mejor a la segmentación de los mercados, nace el movimiento de agrupamiento de marcas. Este proceso es netamente «inflacionista» en términos de marcas, ya que conduce a una multiplicación de las marcas, a un agrupamiento de marcas.

\section{Rol estratégico de los agrupamientos de marcas}

La existencia de límites al territorio de una marca conduce, a las empresas que desean desarrollarse, a crear o adquirir otras marcas a fin de penetrar en los mercados, segmentos o grupos objetivo en los que no podrían penetrar con su actual marca. 
Paralelamente, las restricciones económicas, financieras o de gestión conducen a estas mismas empresas a limitar el número de marcas que gerencian, para maximizar sus inversiones, $y$ para fortalecer $y$ extender los territorios de sus marcas. Esta situación es, pues, total e intrínsecamente esquizofrénica y plantea el tema de la superación y sublimación de un esquema contradictorio.

Numerosas empresas comprenden la importancia de la explotación de múltiples marcas en una misma categoría de productos (Riezebos, 2003: 5), y de esta explotación razonada y organizada obtienen una ventaja competitiva. Así, a lo largo de los años han acumulado un «parque» de marcas, sea por adquisición voluntaria o porque las marcas estaban incluidas en operaciones de acercamiento de las empresas.

Estas marcas han sido agregadas a lo largo del tiempo tal vez de manera incoherente, en el sentido que las respectivas zonas de influencia de cada una de ellas no estaban estrictamente determinadas. Pero la organización de estos agrupamientos de marcas puede representar una ventaja competitiva durable y sostenible basada en los recursos y competencias de la empresa (Trinquecoste, 1995, 1999).

Nuestra proposición es que la marca existe y se proyecta mucho más de lo que los estudios actuales sugieren, puesto que se integra a un portafolio de marcas que participa en la creación y puesta en marcha de la estrategia de la empresa. Si consideramos que el portafolio de marcas emerge del nivel estratégico de la empresa, entonces la caracterización de la relación entre portafolio de marcas y ventaja competitiva deviene un elemento esencial de nuestra investigación. Dado que el portafolio de marcas sobrepasa el nivel de la marca, es su contribución a la creación de la ventaja competitiva estratégica lo que debe ser estudiado (Juga, 1999).

La formalización de los agrupamientos de marcas, es decir, la transformación de una acumulación de marcas en un portafolio de marcas gerenciado de modo proactivo, no ha estado siempre organizada ni pensada en tanto tal por las empresas. Podemos suponer igualmente que las razones de utilización de uno u otro modelo no están exclusivamente ligadas a un enfoque del consumidor. Razones ajenas al marketing pueden tener importancia, como, por ejemplo, la historia, los procesos de adquisición, la filosofía de los gerentes (Laforêt y Saunders, 1999).

El propósito de nuestra investigación es explorar el campo de la estrategia de los portafolios de marcas a nivel de las empresas. Queremos evidenciar la dimensión estratégica de los portafolios de marcas y apoyar el rol mayor de la gestión de un portafolio de marcas como fuente de ventaja competitiva.

Al desplazar las fuentes de ventaja competitiva a un nivel superior (Juga, 1999; Reynaud, 2001), esta ventaja se hace más difícil de comprender y de imitar. Gracias a la combinación de marcas dentro de un portafolio es posible gerenciar de manera sutil y proactiva la elección de productos y la expansión de los territorios de marcas. Por ello, es necesario interrogarse sobre los métodos de creación, desarrollo y utilización de estos portafolios.

¿Existen parámetros y principios que rijan, organicen y regulen la gestión de los 
portafolios de marcas? Cada día las empresas consideran más la gestión del agrupamiento de marcas como un valor clave de su desarrollo o crecimiento. Conviene integrar este metanivel de la marca, ya que puede ser fuente de nuevas ventajas competitivas sostenibles. Desde nuestro punto de vista, se trata de una etapa clave en la comprensión de la ventaja competitiva de numerosas empresas (Sharma, 1999; Slater y Olson, 2001) y del marketing estratégico (Day, 1994; Trinquecoste, 1999).

La reflexión sobre el portafolio de marcas se inscribe en el debate sobre la contribución estratégica del marketing (Day, 1992; Hunt y Morgan, 1995). Muchos modelos de marketing estratégico muestran que los recursos y competencias de la empresa deberían ser el punto de partida de las decisiones estratégicas (Day, 1994; Hunt y Morgan, 1997) y, por lo tanto, preceder a las decisiones de posicionamiento (Juga, 1999).

Para Slater y Olson (2001), la estrategia de la empresa -que ellos denominan «busines strategy»- se relaciona con la creación de la ventaja competitiva, mientras que la estrategia de marketing es el conjunto de decisiones y acciones por las que una empresa trata de alcanzar sus objetivos de marketing y ofrece el valor exigido por el consumidor. La estrategia de marketing se relaciona con las decisiones ligadas a la segmentación del mercado, con la determinación de los grupos-objetivo, con el desarrollo de un posicionamiento fundado sobre las decisiones de producto, de precio, de distribución y de promoción (Slater y Olson, 2001: 1056).

Trinquecoste $(1995,1999)$ utiliza el término de orientación estratégica dual cuando aborda el análisis de la ventaja competitiva de una empresa. Hay dualidad entre una estrategia que preconiza la investigación de una ventaja competitiva basada en el análisis de las potencialidades del mercado y una estrategia orientada a los recursos y competencias de la empresa en sí misma. Esta última opción busca diferenciar la oferta producida focalizándose en la combinación de los recursos y las competencias.

La organización de la gestión de las marcas dentro de la empresa se origina en esta diferenciación fundada sobre la utilización de los recursos y competencias internas, como, por ejemplo, disponibilidad de las marcas y capacidad para gerenciarlas y maximizarlas (poseer las marcas no es una herramienta de diferenciación o de desempeño suficiente).

Las empresas han abandonado los modelos clásicos de planificación y de puesta en práctica de las estrategias ligadas a la gerencia estratégica (en primer lugar General Electric, pionera de la planificación estratégica), para orientarse hacia aproximaciones estratégicas nuevas. En función de su contexto competitivo, numerosas firmas han considerado que la orientación al mercado (Kohli y Jawors$\mathrm{ki}^{5}, 1990$, citados por Sharma, 1999) era una responsabilidad de los dirigentes y que el customer-focus es una política de la organización en su conjunto (MacKenna, 1991). La orientación al mercado se sitúa entonces al nivel de la corporate-level strategy, es decir, al nivel de la gerencia estratégica.

5. Kolhi y Jaworski definen la orientación al mercado como: «the organization-wide generation of market intelligence pertaining to current and future customer needs, disemination of the intelligence across departments, and organizationwide responsiveness to it». 
Este rol subordinado del marketing en la determinación de la estrategia competitiva está en el origen de numerosas discusiones (Day, 1994; MacKenna, 1991; Piercy et al., 1997). Sharma (1999: 74) recuerda el debate sobre el rol del marketing estratégico en el seno de la estrategia competitiva de la firma:

Paralelamente, los imperativos del juego competitivo han restringido a las empresas a observar sus problemas de gestión de los negocios (business problems) de manera «holística» buscando que resolverlas de un modo global (integrative manner) sin preocuparse de asuntos de las fronteras de dominio.

Al mismo tiempo, los investigadores en marketing han comenzado a incorporar los conceptos de la gerencia estratégica, interrogándose sobre el rol de los recursos y competencias en la creación de un desempeño competitivo superior (Day, 1994).

Luego de la declinación de la importancia de la estrategia de marketing, en particular frente a la emergencia del dominio de la gerencia estratégica, Sharma (1999) muestra la emergencia del marketing estratégico como disciplina más integrativa que la estrategia de marketing y la reciente aproximación alrededor del tema de la competitividad entre el marketing estratégico y gerencia estratégica.

El marketing estratégico aparece en respuesta a las críticas según las cuales el marketing ha fracasado en tomar en cuenta correctamente la ventaja competitiva a largo plazo. El marketing estratégico trata de hacer coincidir los recursos de la firma con las oportunidades y restricciones del medio ambiente, a fin de obtener una ventaja competitiva de largo plazo. Así para Day (1994), las organizaciones que logran una ventaja competitiva a través de su política de marketing han desarrollado dos tipos de competencias: una capacidad superior de exploración del mercado o una aptitud para desarrollar una relación duradera con el cliente.

La marca y su gestión son tradicionalmente ilustraciones de estas relaciones/ evoluciones entre el marketing estratégico y la gerencia estratégica. La marca representa una apuesta en el seno de la empresa entre los practicantes del marketing y los responsables de marketing, en particular como fuente de creación de valor (Farqhar, 1990; Aaker, 1991; y Kapferer, 1991).

Pero hoy día, la intensidad del juego competitivo, el desarrollo de nuevos mercados, las extensiones, el crecimiento internacional y muchos otros factores hacen que numerosas compañías hayan acumulado gran cantidad de gamas de productos y de marcas diferentes.

Una cuestión central de la empresa no es sólo la gestión de la marca en sí, sino, más bien, la organización de las relaciones entre las marcas al interior de la empresa y la existencia de una relación jerárquica entre estas marcas. Esto origina el desarrollo de portafolios de marcas, a fin de prevenir los efectos de canibalización entre marcas, la gestión de la relación entre estas marcas y el equilibrio del portafolio de marcas (Riezebos, 2003).

¿Cuál es la lógica y cuáles los criterios principales en esta modificación del nivel estratégico de la marca y del desplazamiento de esta dimensión estratégica hacia un nivel superior de agrupamien- 
to, el portafolio de marcas? ¿Existen puntos comunes según la estructura de las empresas o los mercados donde éstas compiten, o bien las decisiones son circunstanciales, no modelables y no extrapolables, incluso ante situaciones similares?

Paradójicamente, los trabajos sobre este asunto son escasos. Hay muchas investigaciones respecto a la marca en sí misma, pero es sorprendente constatar el escaso número de trabajos relativos a la marca dentro del contexto estratégico de una metaestructura: el portafolio de marcas.

El portafolio de marcas corresponde perfectamente a la perspectiva del marketing estratégico diseñado por Sharma (1999) y Trinquecoste (1999). En efecto, creemos que es posible salir de la contradicción que, por un lado, conduce a multiplicar las marcas y, por el otro, a limitarlas. Se trata de una percepción paradigmática nueva hacia el portafolio de marcas, según la cual el valor de la marca se establece en función del dimensionamiento relativo de su territorio respecto a las otras marcas de la misma empresa.

El objetivo de las empresas, en particular las más grande, es ser capaces de «salir por arriba» de la contradicción enunciada anteriormente (multiplicación de marcas versus limitación de marcas) por una proyección estratégica, desplazando el nivel de la ventaja competitiva (Trinquecoste, 1999) para hacerla más difícil de imitar (Reynaud, 2001).

Este proceso pasa por una gestión activa de los agrupamientos de marca, es decir, por la selección de ciertas marcas sobre las que estas empresas van a basar su desarrollo ulterior y por la delimitación anticipada de los territorios respectivos de cada una de las marcas que componen el portafolio.

Esto explica por qué llamaremos gestión del portafolio de marcas al proceso consistente en organizar las relaciones entre marcas dentro de una misma empresa.

\section{Definición y dimensiones del portafolio de marcas}

El concepto de portafolio de marcas implica que la gestión organizada del todo sea superior a la gestión autónoma de las unidades individuales, que son las marcas (Riezebos, 2003).

Las tipologías clásicas de la marca (Aaker, 1991; Kapferer, 1991; Lambin, 1994; Laforêt y Saunders, 1999) no distinguen explícitamente el caso del portafolio de marcas. Sin embargo, caracterizan las marcas sobre la base de las interrelaciones entre ellas. Pero de marca-paraguas a marca-producto o marca-endosadora (endorser brand), las diferencias residen esencialmente en el número de niveles de distancia de la marca al producto. Cuando se aborda la relación intermarcas sólo se analiza desde el punto de vista de una relación de subordinación (el caso de las marca-endosadoras).

La gestión de agrupamientos de marca implica, a la vez, una elección de marketing y una elección estratégica. Elección de marketing, porque se trata de determinar las variables de diferenciación de las marcas entre ellas dentro de una misma empresa e igualmente frente al consumidor. Elección estratégica, porque se trata de asignar los medios humanos y financieros; recursos y competencias entre las 
diferentes marcas pero, sobre todo, de determinar las áreas de influencia respectiva de las marcas. Esta doble elección implica un fenómeno complejo difícil de describir y reconstruir.

A fin de hacer operacionalizable el concepto de portafolio de marcas y de medir su valor paradigmático es indispensable proponer una definición de este objeto de investigación. En función de los desarrollos enunciados en los dos párrafos anteriores, proponemos la siguiente definición:

Llamaremos portafolio de marcas a todo el esquema de gestión activa de los agrupamientos de marcas; es decir, a la selección de ciertas marcas sobre las que la empresa va a focalizar su desarrollo ulterior, así como a la organización estructurada de las relaciones de estas marcas entre ellas. En ausencia de una definición común, sugerimos, a partir de los escasos trabajos existentes sobre este concepto (Barwise y Robertson, 1993; Riezebos, 2003), la definición operacional siguiente:

Un portafolio de marcas representa la fase superior del agrupamiento de marcas dentro de una firma y corresponde a una gestión activa de las relaciones entre las marcas dentro de esta firma.

Será necesario hacer mensurable este fenómeno en el futuro. Con este propósito analizaremos las diferentes dimensiones de esta definición:

- «Fase superior del agrupamiento de marcas» El portafolio es la resultante de una trayectoria histórica. En este sentido, para que haya un portafolio es necesaria una acumulación, es indispensable que haya más de una marca dentro de la firma. Pero esta condición no es suficiente, también se necesita que haya la voluntad estratégica de superar la simple yuxtaposición para gerenciar la relación entre esas marcas. Ello implica que la relación entre marcas merezca reflexión, decisión y sea fuente de asignación de medios dentro de la empresa.

- «Gestión activa de las relaciones» El asunto del portafolio se plantea cuando el agrupamiento de marcas no basta para las necesidades de desarrollo de la relación entre la empresa y sus consumidores. La gestión no puede ser realizada sólo por los responsables de marca, pues supone un proceso de arbitraje, coordinación y elección. Será pues en el nivel de la gerencia estratégica que se hará la gestión activa de las relaciones entre marcas.

- «Relaciones entre marcas dentro de una firma» La relación entre marcas está en el corazón del concepto de portafolio. El lugar relativo de una marca respecto a otra dentro del portafolio se diseña en función de los límites de los territorios de marcas, de su potencial de extensión y de consideraciones de política de la empresa.

Estas tres dimensiones de la estrategia del portafolio de marcas nos permiten realizar una primera etapa para evaluar el contenido de una estrategia de portafolios de marcas, cuyos riesgos y condiciones de operacionalización hemos resumido.

\section{Conclusión}

El reconocimiento creciente de las marcas como fuente de ventaja competitiva 
sostenible acentúa la importancia de los modelos conceptuales sobre las estrategias de marcas (Louro y Cunha, 2001). El aporte del análisis de portafolio es poner en evidencia, a un nivel superior, principios directores nuevos.

Cada día, el portafolio de marcas es una herramienta más elemental, en la medida que permite superar la cuestión competitiva a nivel de la marca y sustituirla por una metadimensión susceptible de crear una ventaja competitiva durable y sostenible.

Desplazando la ventaja competitiva al nivel superior del portafolio, ésta se hace más difícil de comprender y de imitar. Crea, entonces, una nueva barrera de ingreso.

El presente artículo sitúa el contexto -límites de las extensiones de marca y del territorio de marca- y las etapas -agrupamiento de marcas- que conducen a la constitución de un portafolio de marcas. Hemos desarrollado el rol y la importancia estratégica decisiva y actual de este concepto y propuesto una definición del portafolio de marcas que hace operacionalizable el concepto y establece las bases para futuras investigaciones centradas sobre la ventaja competitiva que ella nos procura. 


\section{Referencias bibliográficas}

AAKER, D. 1991. Managing Brand Equity: Capitalizing on the Value of a Brand Name. Nueva York: The Free Press.

AAKER, D. y KELLER, K. 1990. Consumer evaluations of brand extensions. Journal of Marketing. Vol. 54, n. ${ }^{\circ}$, págs. 27-33.

AAKER, J. L. 1997. Dimensions of brand personality. Journal of Marketing Research. Vol. 34, págs. 347-356.

ALBA, J. W. y CHATTOPADHYAY, A. 1986. Salience effects in brand recall. Journal of Marketing Research. ․o 23, págs. $363-$ 369.

AMINE, A. 1998. Consumers' true brand loyalty: the central role of commitment. Journal of Strategic Marketing. N. ${ }^{\circ}$, págs. 305-319.

AZOULAY, A. 2002. Las échelles de personnalité de marca mesurent-ellas réellement la personnalité. Journée AFM Las Marcas. Dic., págs. 1-31.

BARWISE, P. y ROBERTSON, T. 1993. Brand portfolios. European Management Journal. Vol. 10, n. ${ }^{\circ}$ 3, págs. 277-285.

BENSA, F. y LADWEIN, R. 1996. Le choix de la marque, le capital-marque et l'activité comportementale face au linéaire. Les cahiers de la recherche de l'IAE, Lille.

BOTTOMLEY, P. A. y HOLDEN, S. 2001. Do we really know how consumers evaluate brand extensions? Empirical generalizatio$\mathrm{ns}$ based on secondary analysis of eight studies. Journal of Marketing Research. N. ${ }^{\circ} 38$, págs. 494-500.

BRONIARCZYK, S. y ALBA, J. W. 1994. The importance of brand in brand extension. Journal of Marketing Research. Vol. 31, n. ${ }^{\circ}$ 5, págs. 214-228.

CAPRARA, G. V.; BARBARANELLI, C. y GUIDO, G. 2002. La personnalité de marque: la métaphore est-elle appropriée? Recherches et Applications Marketing. Vol.17, n. ${ }^{\circ} 1$, págs. 75-88.

CEGARRA, J. J. 1994. Gérer un portefeuille de marques. Décisions Marketing. $\mathrm{N}^{\circ} 3$, págs. 81-91.

CEGARRA, J. J. y MERUNKA, D. 1993. Les extensions de marques: concepts et modèles. Recherche et Applications Marketing. Vol. 8, n. ${ }^{\circ} 1$, págs. 53-76.

DACIN, P. A. y SMITH, D. C. 1994. The effect of brand portfolio characteristics on consumer evaluations of brand extensions. Journal of Marketing Research. N. ${ }^{\circ} 31$, págs. 229-242.

DAY, G. S. 1994. The capabilities of marketdriven organizations. Journal of Marketing. Oct., vol. 58, págs. 37-52.

DYSON, P.; FARR, A. y NIGEL, S. H. 1996. Understanding, measuring and using brand equity. Journal of Advertising Research. Vol. 36, n. ${ }^{\circ}$ 6, págs. 9-21.

EVRARD, Y.; PRAS, B. y ROUX, E. 2000. Market. París: Dunod. 
FARQHAR, P. 1990. Managing brand equity. Journal of Advertising Research. Vol. 30, n. ${ }^{\circ} 4$, págs. 7-12.

FERRANDI, J. M. y VALETTE-FLORENCE, P. 2002. Le transfert d'une échelle de personnalité humaine réduite à la marque. Actes de la Journée AFM-Les Marques. Dic.

FOURNIER, S. 1998. Consumers and their brands: Developing relationship theory in consumer research. Journal of Consumer Research. Vol. 24, págs. 343-373.

GÜRHAN-CANLI, Z. y MAHESWARAN, D. 1998. The effects of extensions on brand name dilution and enhancement. Journal of Marketing Research. N. 35, págs. 464-473.

GURVIEZ, P. 1999. La confiance comme variable explicative du comportement du consommateur: proposition et validation empirique d'un modèle de la relation à la marque intégrant la confiance. Actes de la Conférence de l'AFM. Strasbourg, págs. 301-328.

HERR, P.M.; FARQUHAR, P. H. y FAZZIO, R.H. 1996. Impact on dominance and relatedness on brand extensions. Journal of Consumer Psychology. Vol. 5, n. ${ }^{\circ}$ 2, págs. 135-159.

HUNT, S. y MORGAN, R. 1995. The comparative advantage theory of competition. Journal of Marketing. Vol. 59, n. ${ }^{\circ}$ 2, págs. 1-15.

1997. Resource-advantage theory: a snake swallowing its tail or a general theory of competition. Journal of Marketing. Oct., n. ${ }^{\circ} 61$, págs. 74-82.
JACOBY, J. y CHESTNUT, R. 1978. Brand loyalty and measurement. Nueva York: John Wiley.

JUGA, J. 1999. Generic capabilities: combining positional and resource-based views for strategic advantage. Journal of Strategic Marketing. Vol. 7, págs. 3-18.

KAPFERER, J. N. 1991. Les marques capital de l'entreprise. París: Les Editions d'Organisation.

— 2000. Re marques. París: Les Editions d'Organisation.

KAUFMAN-ODIN, N. 1997. La typicalité: une heuristique des choix de marque. Thèse de gestion.

KELLER, K. 1993. Conceptualizing, measuring and managing customer-based brand equity. Journal of Marketing. N. ${ }^{\circ}$ 57, págs. $1-22$.

KLINK, R. R. y SMITH, D.C. 2001. Threats to the external validity of brand extension research. Journal of Marketing Research. N. ${ }^{\circ} 38$, págs. 326-335.

KOHLI, A. K. y JAWORSKI, B. 1990. Market orientation: the construct, research and propositions and management implications. Journal of Marketing. N. ${ }^{\circ}$ 54, págs. 1-18.

KOTLER, P. y DUBOIS, B. 1997. Marketing Management. 9a ed. París: PubliUnion.

KRISHNAN, H. S. 1996. Characteristics of memory associations: a consumer-based brand equity perspective. International Journal of Research in Marketing. N. ${ }^{\circ} 13$, págs. 389-405. 
LACOEUILHE, J. 2000. L'attachement à la marque: proposition d'une échelle de mesure. Recherche et Applications en Marketing. Vol. 15, n. ${ }^{\circ}$, págs. 61-77.

LADWEIN, R. 1993. Extension de marques et catégories cognitives. Thèse de doctorat en Sciences de Gestion, IAE de Lille.

LAFORET, S. y SAUNDERS, J. 1994. Managing brand portfolios: How the leaders do it. Journal of Advertising Research. Set.-oct., vol. 34, n. ${ }^{\circ}$ 5, págs. 64-77.

1999. Managing brand portfolios: why leaders do what they do? Journal of Advertising Research. Feb., págs. 51-66.

LAMBIN, J. J. 1989. La marque et le comportement de choix de l'acheteur. En Kapferer, J. N. y Thoenig, J. C. La marque: moteur de la compétitivité des entreprises et de la croissance économique. McGraw Hill.

LANCASTER, K. J. 1966. A new approach to consumer theory. Journal of Political Economy. Vol. 74.

LEVY, S. J. 1959. Symbols for sale. Harvard Business Review. Summer, vol. 37, págs. 117-124.

LOKEN, B. y JOHN, D. R. 1993. Diluting brand beliefs: When do brand extensions have a negative impact? Journal of Marketing. N. ${ }^{\circ} 57$, págs. 71-84.

LOURO, M. y CUNHA, P. 2001. Brand management paradigms. Journal of Marketing Management. N. ${ }^{\circ} 17$, págs. 844-878.

MAC ENALLY, M. y DE CHERNATONY, L. 1999. The evolving nature of branding: consumer and managerial considerations.
Journal of Consumer and Market Research. 99, 02.

MACKENNA, R. 1991. Marketing is everything. Harvard Business Review. Ene.-feb., n. ${ }^{\circ} 69$, págs. 65-79.

MICHEL, G. 2000. La stratégie d'extension de marque. París: Vuibert.

MILBERG, S. 1993. Reciprocity effects of brand extensions: Dilution, fortification, expansion. Ph.D. dissertation, University of Pittsburg.

MORRIN, M. 1999. The impact of brand extensions on parent brand memory, structures and retrieval processes. Journal of Marketing Research. N. ${ }^{\circ}$ 36, págs. 517-525.

PERRIN-MARTINENCQ, D. 2002. Proposition d'une échelle de mesure du détachement de la marque. Actes de la Journée AFM Les Marques. Dic., págs. 1-23.

PIERCY, N.; LLOYD, C.; PETERS, L. y LANE, N. 1997. Marketing management, market strategy and strategic management: Domain realignment and redefinition. Journal of Strategic Marketing. N. ${ }^{\circ} 5$, págs. 50-62.

PLUMMER, J. T. 2000. How personnality makes a difference. Journal of Advertising Research. N. ${ }^{\circ}$ 24, págs. 27-31.

REYNAUD, E. 2001. Compétences centrales: Premier pas vers une définition opérationnelle. Xème conférence de l'AIMS, Laval.

RIES, A. y RIES, L. 2000. Les 20 lois du capital marque. París: Dunod. 
RIES, A. y TROUT, J. 1981. Positionning: the battle for your mind. Nueva York: Mac Graw Hill.

RIEZEBOS, R. 2003. Brand Management, a Theoretical and Practical Approach. Harlow: Prentice Hall.

SAPPINGTON, D. y WERNERFELT, B. 1985. To brand or not to brand? Journal of Business. Jul., n. ํ 58, págs. 279-293.

SEMPRINI, A. 1992. Le marketing de la marque: approche sémiotique. París: Liaisons.

SHARMA, S. 1999. Trespass or symbiosis? Dissolving the boundaries between strategic marketing and strategic management. Journal of Strategic Marketing. Vol. 7, págs. 73-88.
SICARD, M. C. 2001. Ce que marque veut dire... París: Editions d'Organisation.

SLATER, S. y OLSON, E. 2001. Marketing's contribution to the implementation of business strategy: an empirical analysis. Strategic Management Journal. Vol. 22, págs. 1055-1067.

SWAMINATHAN, V.; FOX, R. J. y REDDY, S. K. 2001. The impact of brand extension introduction on choice. Journal of Marketing. $\mathrm{N}^{\circ}{ }^{\circ} 65$, págs. 1-15.

TRINQUECOSTE, J. F. 1995. Marketing stratégique. Encyclopédie de gestion. Págs. 194-197.

- 1999. Pour une clarification théorique du lien marketing-stratégie. Recherche et Applications en Marketing. Vol. 14, n. ${ }^{\circ}$ 1/99, págs. 59-80. 